\title{
Sensitivity to negative feedback among children and adolescents: An ERP study comparing developmental differences between high-worriers and low-worriers
}

\author{
Taylor Heffer ${ }^{1} \cdot$ Teena Willoughby $^{1}$ \\ Published online: 28 April 2020 \\ (C) The Psychonomic Society, Inc. 2020
}

\begin{abstract}
Neurodevelopmental imbalance models suggest that asynchrony in the maturation of interconnections between brain regions contributes to adolescents being more sensitive to emotionally salient events (e.g., negative feedback) than children. There may, however, be important individual differences to consider when investigating sensitivity to negative feedback. For example, worriers tend to have a greater sensitivity to negative feedback than low-worriers. Thus, it may be that adolescents' sensitivity to negative feedback is tied to worry. One way to test this question is to compare worriers to nonworriers separately for both children and adolescents. If only adolescent worriers are sensitive to negative feedback (i.e., low-worriers are not), then sensitivity to negative feedback may be linked to higher rates of worry. If however, adolescent nonworriers also have a sensitivity, then adolescents in general may be sensitive to negative feedback. The current study $(N=100$, Mage $=11.26$, standard deviation $=$ 1.71) used event-related potentials (ERPs) to investigate neural differences in sensitivity to negative feedback among adolescents and children with high and low levels of worry. For both children and adolescents, worriers had a larger P3 amplitude to negative feedback than nonworriers. This difference, however, was smaller among the adolescents (i.e., adolescent nonworriers also had a large $\mathrm{P} 3$ amplitude to negative feedback). Our results support neurodevelopmental imbalance models that suggest adolescents in general are sensitive to emotionally salient events, such as receiving negative feedback.
\end{abstract}

Keywords Event-related potentials $\cdot$ Adolescent $\cdot$ P $3 \cdot$ Worry $\cdot$ Children $\cdot$ Sensitivity to negative feedback

Adolescence often is considered a transitional period marked by physical, psychological, and social changes (Spear, 2000). One notable change is the increase in adolescents' sensitivity to emotionally salient events (e.g., sensitivity to negative feedback). Indeed, compared with children, adolescents tend to report more sensitivity to negative feedback (O'Brien \& Bierman, 1988; Vervoort et al., 2010; Westenberg, Drewes, Goedhart, Siebelink, \& Treffers, 2004). For example, O'Brien and Bierman (1988) found that adolescents (grade 8) were more likely than children (grade 5) to report that rejection impacted their sense of self-worth. Furthermore,

Electronic supplementary material The online version of this article (https://doi.org/10.3758/s13415-020-00791-8) contains supplementary material, which is available to authorized users.

Taylor Heffer

th10ww@brocku.ca

1 Department of Psychology, Brock University, 1812 Sir Isaac Brock Way, St. Catharines, ON L2S 3A1, Canada
Westenberg et al. (2004) found that fear of negative social evaluation was higher among adolescence compared with children (age range in the study was 8 to 19). Although these studies highlight social negative feedback (e.g., rejection), sensitivity to negative feedback also includes an emotionally salient event, such as receiving negative feedback about performance.

Recently, a number of neurodevelopmental imbalance models have been used to help explain why adolescents in general (i.e., not just in social settings) - compared with children - may be more sensitive to emotionally salient experiences, such as receiving negative feedback (Casey, 2015; Somerville, Jones, \& Casey, 2010; Steinberg, 2008). According to these models, adolescence behaviour may be affected by an imbalance between an early maturing limbicstriatal system (possibly related to puberty), associated with affective processing, and a slower developing prefrontal cortex system, associated with cognitive control. This asynchrony is thought to lead to heightened activation of the limbicstriatal region during early to mid-adolescence when neural connections to the prefrontal cortex that might dampen the 
activation (if appropriate) are not fully mature, thus making this age group more sensitive to emotionally salient stimuli compared with children.

In line with these theories, studies have found that subcortical regions (e.g., the amygdala) increase in volume across puberty (Goddings et al., 2014) and mature earlier than higher-order cortices (e.g., prefrontal cortex; Galvan et al., 2006; Gogtay et al., 2004; Mills, Goddings, Clasen, Giedd, \& Blakemore, 2014). Adolescents, compared with children, also have heighted activation in limbic regions when viewing negative faces (Hare et al., 2008) and when receiving negative feedback (Bolling et al., 2011; Moor, van Leijenhorst, Rombouts, Crone, \& van der Molen, 2010). Thus, there has been some work suggesting that adolescents may have greater neural sensitivity to negative feedback than children. At the same time, there may be important individual differences to consider when investigating sensitivity to negative feedback. For instance, adolescent worriers report greater sensitivity to negative feedback compared with adolescents with low levels of worry (Balle, Tortella-Feliu, \& Bornas, 2013). Studies using event-related potentials (ERPs) also have found that worriers have greater P3 activation (an ERP component that is typically larger when an individual is paying more attention to the feedback; Huang et al., 2015; Luck, 2005) to negative feedback compared with low-worriers (De Pascalis, Strippoli, Riccardi, \& Vergari, 2004; Miltner et al., 2005; Sewell, Palermo, Atkinson, \& McArthur, 2008; although see BarHaim, Lamy, \& Glickman, 2005 for a study that found no differences in the P3 between individuals with high and low anxiety). Thus, worriers tend be more sensitive to negative feedback than low-worriers. Of note, however, these ERP studies have primarily focused on university students.

There is a paucity of research investigating whether individual differences in worry among adolescents and children might affect their sensitivity to negative feedback using the P3. In a sample of adolescents, Reeb-Sutherland et al. (2009) found a trend whereby high sensitivity to negative feedback and larger P3 amplitudes was associated with greater anxiety. Beyond that, little work that investigated not only individual differences (e.g., worry) in adolescents' neural sensitivity to negative feedback, but also how adolescents compare to children.

It may be that adolescents' sensitivity to negative feedback is tied to worry. In this case, we would expect only adolescents who report higher levels of worry to have a larger P3 amplitude to negative feedback - not adolescents who report low levels of worry. The imbalance neurodevelopmental models, in contrast, might suggest that adolescents in general likely are sensitive to emotionally salient stimuli; that is, in the heat of the moment - directly after receiving negative feedbackboth adolescent worriers and low-worriers might show sensitivity to the feedback. Thus, adolescents who report low levels of worry are a key group of interest in this study.

\section{The Current Study}

The goal of this ERP study was to investigate whether adolescents and children with high versus low levels of worry differ in their sensitivity to negative feedback (when receiving lossfeedback about their performance on a task). We had three main research questions: (1) Do adolescents have a greater neural sensitivity to negative feedback than children (main effect of age group)?; (2) Do worriers have greater neural sensitivity to feedback than low-worriers (main effect of worry)?; (3) Do worriers and low-worriers show similar sensitivity to negative feedback only in adolescence but not in childhood (interaction between worry and age group)?

Overall, we expect that adolescents will have a greater P3 amplitude to negative feedback than children, and worriers will have a greater P3 amplitude to negative feedback than low-worriers. In terms of the interaction, given the lack of research in this area, this analysis is more exploratory. It may be that adolescents' sensitivity to negative feedback is tied to worry, or as the neural developmental models might suggest, it may be that adolescents in general are sensitive to negative feedback. It also is not clear whether adolescent worriers will have even larger P3 amplitudes than adolescent lowworriers. In terms of the children, if worry is associated with greater attention to negative feedback (in line with ERP studies using university students), then we would expect that only children who are worriers will have a large P3 amplitude to negative feedback compared with children who are low worriers.

We also were interested in comparing how the results might differ depending on whether pubertal status or grade-level is used to classify adolescents versus children. Importantly, neurodevelopmental imbalance models highlight that puberty might be a key reason for the brain changes that occur in adolescence (Casey, 2015; Somerville et al., 2010; Steinberg, 2008). Furthermore, previous research has found that pubertal development is a better marker than age (van den Bos, de Rooij, Miers, Bokhorst, \& Westenberg, 2014). Thus, another goal of our study was to test whether there were any differences in the results when using grade versus pubertal status to distinguish between children and adolescents. We conducted the analyses first using grade and then again using pubertal status as a way to identify any differences between these approaches. Critically, because theory and previous research highlight the importance of puberty, we expect that pubertal development will be a more sensitivity measure than grade status.

Although we were primarily interested in group differences in sensitivity to negative feedback, we also provided participants with positive feedback during our task. Thus, while worriers may be particularly concerned with negative feedback, neurodevelopmental models suggest that adolescents may be sensitive to emotionally salient events in general 
(e.g., both negative and positive feedback). As a secondary analysis, we investigated whether groups differed in their sensitivity to positive feedback (see supplemental materials).

\section{Method}

\section{Participants}

The current sample included 127 students (50.4\% female; age range: $8-14 ;$ Mage $=11.26$, standard deviation $[\mathrm{SD}]=1.71$ ) from several elementary and high schools in southern Ontario, Canada. Students were part of a larger study examining the relationship between wellbeing and youth health-risk behaviours. Parents were asked to identify whether their child had any illnesses or disabilities (either physical or mental). One participant was excluded from the study based on a diagnosis of autism. Parent report indicated that $87.2 \%$ of the children and adolescents were white, $2.6 \%$ were Hispanic, $0.9 \%$ were black, and $8.5 \%$ were mixed (an additional $0.9 \%$ of parents indicated that they preferred not to answer the question). Mean levels of parental education fell between "some college, university, or apprenticeship program" and "completed a college/ apprenticeship and/or technical diploma."

\section{Procedure}

Students were invited to participate in the study through visits to schools. Surveys were completed in classrooms during school hours and all participants received gifts (e.g., backpacks) as compensation. Participants also completed a Mobile Lab component where they each played computer tasks on their own while EEG was recorded. There were 12 participants who did not fill out the worry scale; therefore, they were not included in this study. Six participants did not complete the task due to equipment issues, and eight participants were not included because their ERP data was not usable (e.g., contained a large number of muscle/movement artifacts). Thus, the final sample included 100 participants. The University Ethics Board approved this study. Participants provided informed assent, and their parents provided informed consent.

\section{Primary Measure}

Worry Participants reported the extent to which they agreed with three items examining worry ("I know I should not worry about things but I just cannot help it"; "I worry about getting in trouble"; "I worry about making mistakes") on a scale ranging from 1 (Almost Never) to 4 (Almost Always). Higher scores indicated higher levels of worry. Cronbach's alpha was 0.844 .
Age Group To distinguish between children and adolescents based on age group, anyone in grades 3 to 5 was considered a child (Mage $=9.627, S D=0.618)$, and anyone in grades 6 to 8 was considered an adolescent $($ Mage $=12.404, S D=1.100)$.

Pubertal Status Pubertal status was assessed using the Puberty Development Scale (PDS; Petersen, Crockett, Richards, \& Boxer, 1988). The PDS assesses body hair, facial hair, and voice development in boys, and body hair, menarche, and breast development in girls. All items were rated on a 4point scale from 1 (not yet started changing) to 4 (change seems complete). For boys, their scores were summed such that any score of 5 or lower (with no 3-point responses) were considered pre/early puberty, while a score of 6 or more was considered mid-later puberty (Carskadon \& Acebo, 1993). For girls, a score of 3 or less, without menarche, was categorized as pre-early puberty, while a score of 3 or more, plus a yes to menarche, indicated mid-late puberty (see Carskadon \& Acebo, 1993 for scoring scheme). The PDS scale exhibits good reliability and validity (Carskadon \& Acebo, 1993; Petersen et al., 1988).

Balloon Analogue Risk Task The Balloon Analogue Risk Task (BART) is a behavioural task that has been used to measure risky decision-making (Lejuez et al., 2002). Traditionally, participants are instructed to inflate a series of balloons to earn points. The goal is to pump each balloon up as much as possible as each pump incrementally adds points for that trial. As the balloon gets larger, however, it is more likely to pop, in which case the participants lose the points that they accumulated on that trial (Lejuez et al., 2002). They still keep the points they received on the other trials. Given that this task provides feedback associated with losing (i.e., when the balloon pops and points are lost) and winning (i.e., when the balloon does not pop and points are won), it facilitates the examination of sensitivity to negative feedback as well as sensitivity to positive feedback using ERPs (Chandrakumar, Feuerriegel, Bode, Grech, \& Keage, 2018; Fein \& Chang, 2008; Gu, Zhang, Luo, Wang, \& Broster, 2018; Takács et al., 2015).

In order to use the BART for an ERP study, there were important modifications to make to the task. First, studies using the BART often allow participants to inflate the balloon at their own pace (Fein \& Chang, 2008; Gu et al., 2018; Kessler, Hewig, Weichold, Silbereisen, \& Miltner, 2017; Kiat, Straley, \& Cheadle, 2016; Takács et al., 2015; Webber, Soder, Potts, Park, \& Bornovalova, 2017; Xu et al., 2016). One limitation associated with allowing participants to sequentially pump the balloon at their own pace is that researchers are unable to time-lock the ERP to the exact moment participants decide that they are going to cash out. In other words, the researchers are unable to time-lock the ERP to the "win" feedback, because the point at which the participant 
decides they are going to cash out is not identifiable. To address this concern, we had participants choose the number of pumps that they wanted to inflate the balloon at the beginning of the trial (Euser et al., 2013; Pleskac, Wallsten, Wang, \& Lejuez, 2008; Yau et al., 2015). Participants then observed the balloon as it either safely reached the inflation number they picked (i.e., they won the points for that trial), or the balloon burst before reaching that point (i.e., they lost the points for that trial). Participants in this case do not know that they have won points during the trial until they receive feedback - making feedback salient for both wins and losses. This approach allowed us to time-lock the ERPs to the exact moment the participant receives feedback during that trial.

Another limitation that is important to address before using the BART for an ERP study is the feedback stimulus used in the task. In contrast to the win feedback, the loss feedback often is an exploding balloon, while the win feedback consists of a balloon with text in the middle or just a screen informing the participants of the win (Euser et al., 2013; Fein \& Chang, 2008; Gu et al., 2018; Kessler et al., 2017; Kiat et al., 2016; Kóbor et al., 2015; Xu et al., 2016). Therefore, it is difficult to disentangle whether participants are sensitive to the feedback itself or if they are just more sensitive to a startling explosion. To address this concern, we modified the task to ensure that the stimulus for wins and losses were comparable (i.e., similar feedback was given for both wins and losses). Specifically, for both win and loss feedback, we made the text, font, and balloon size consistent, and both feedback messages were written inside of the balloon. We also made sure that the loss feedback was no longer a startling explosion but instead depicted a balloon with a few marks in it to represent that it had popped. This modification ensured that sensitivity to loss would not be driven by the stimulus used to provide the feedback (e.g., a startling explosion). Overall, these modifications allowed us to directly compare sensitivity to wins and sensitivity to losses without concern that results would be confounded by the stimulus or by not being able to examine feedback to wins in the same way as losses.

The task consisted of 90 trials with a maximum breaking point of 20 pumps. The probability of the balloon popping increased as the number of pumps chosen increased (e.g., choosing to pump the balloon up to " 15 " had a greater likelihood of it popping compared with pumping the balloon up to "5"). After feedback was presented, a new balloon appeared after 1,000 ms. Participants earned one point for every pump of the balloon, and points for all the "win" trials were summed to calculate their total points. Participants were instructed that the goal of the task was to earn as many points as possible.

\section{Electrophysiological Recording}

Electroencephalography (EEG) was recorded continuously from a BioSemi ActiveTwo system using a 96-channel montage and 7 face sensors. The data were digitized at a sampling rate of $512 \mathrm{~Hz}$. Our pre-processing pipeline identify scalp channels, time course activations, and independent components that represented unreliable and non-stationary signals.

\section{Pre-processing (Channels)}

Pre-processing was automated (using MATLAB 2012b scripts) to be performed using EEGLAB (Delorme \& Makeig, 2004) version 13.6.5b and was then executed using Octave on Compute Canada's high performance computer cluster (Cedar; see Desjardins \& Segalowitz, 2013; van Noordt, Desjardins, \& Segalowitz, 2015; van Noordt, Desjardins, Gogo, Tekok-Kilic, \& Segalowitz, 2017 for more details). The data were first separated into 1 -second nonoverlapping time windows. For each time window, the voltage variance across each channel was calculated (a 20\% trimmed mean was used). Channels were flagged as unreliable if they had a z-score six times greater than the voltage variance across all channels. Time-periods (i.e., the 1-second time windows) were considered unreliable if more than $10 \%$ of the channels were identified as having extreme voltage variances. Finally, any channels that were flagged in more than $20 \%$ of the timeperiods were considered unreliable throughout the recording.

To minimize spatial bias introduced by variance in channel artifacts across subjects, we used an interpolated average reference procedure. Channels containing clean signal are used to interpolate to 19 spatially balanced sites arranged in the 10 20 layout. The average of these 19 interpolated sites are used as the reference and subsequently subtracted from each of the original channels containing clean signal. The data were filtered with a $1-\mathrm{Hz}$ high pass and $30-\mathrm{Hz}$ low pass filter given that cortical activity would not be expected to exceed $30 \mathrm{~Hz}$. After this step, the data were again checked for the same issues reported above: (1) channels that are unreliable within a given time-period; (2) time-periods that are unreliable; (3) and channels that are unreliable throughout the recording. Specifically, any channels that were unlike its neighbouring channels (e.g., had a low correlation with channels around it) were flagged. A channel was flagged as unreliable if it had a z-score that was 2.326 times greater than the mean of the $20 \%$ trimmed distribution of correlation coefficients. Time-periods were considered unreliable if more than $10 \%$ of the channels within the window were flagged as unreliable. Any individual channels that were flagged in more than $10 \%$ of time-periods were considered unreliable across the entire recording. Bridged channels (i.e., channels that are highly correlated with invariable signal) were identified after dividing the average maximum correlation by the standard deviation of the distribution of correlation coefficients. Channels that had a positive zscore that was eight times greater than the $40 \%$ trimmed distribution of coefficients were flagged as bridged channels. 


\section{Pre-Processing (Components)}

After pre-processing the channel data, all data (channels and time periods) that had not been flagged as unreliable was concatenated back into continuous data. These data were then submitted to an initial Adaptive Mixture of Independent Component Analysis (AMICA) to identify different components of the EEG data (e.g., heart rate components, cortical components etc.). This process helps to separate brain activity (neural components) from nonneural activity (e.g., eye blinks).

During this procedure, the data were windowed into 1second time epochs. Unreliable components were detected by comparing each individual component to the variance among all components. Components were flagged if they had a z-score that was 2.326 times greater than the trimmed mean. Time-periods that had more than $10 \%$ of its components flagged were considered unreliable. The data were then concatenated into the continuous time course and submitted to three simultaneous AMICA decompositions to assess whether components were replicable (i.e., is muscle movement consistently being classified as muscle movement when the process is repeated multiple times). The procedure above for identifying unreliable components (within 1-second epochs) was completed again using the continuous time series data. Next, a dipole (which identifies the position and orientation for the distribution of positive and negative voltages) was fit using the dipfit plugin in Matlab (Oostenveld, Fries, Maris, \& Schoffelen, 2011). Components with a dipole fit residual variance greater than $15 \%$ were flagged. Finally, components were classified using the ICMARC plugin. This process assesses each component against a crowd-sourced database to identify activation consistent with five different categories: eye blinks, neural, heart, lateral eye movements, muscle contamination, and mixed signal.

After pre-processing, a quality control review was completed to ensure that the decisions made during pre-processing were appropriate. This procedure was completed by one trained research assistant who assessed the accuracy of the independent component classifications. For example, the research assistant would identify whether cortical components were correctly distinguished from noncortical components (e.g., muscle, eye blinks, etc.) based on topographical projection, continuous activation, dipole fit, and power spectrum profile. Thus, the quality control review involved using the independent components to help with artifact correction (see Table 1 for summary results of the artifact procedure).

\section{EEG post-processing}

EEG data were then segmented into single trials and timelocked to the onset of the win/lose BART feedback stimuli. Epochs ( -200 to $600 \mathrm{~ms}$ ) were extracted to feedback onset
Table 1. Means and standard deviations resulting from the artifact detection procedure

\begin{tabular}{lll}
\hline Artifact category & Mean (\%) & SD (\%) \\
\hline Time & & \\
Extreme voltage variance & 1.93 & 1.84 \\
Low channel correlation & 0.13 & 0.29 \\
ICA variance 1 & 8.46 & 5.56 \\
ICA variance 2 & 1.75 & 1.60 \\
All methods & 12.26 & 7.92 \\
Channels & & \\
Extreme voltage variance & 2.28 & 1.93 \\
Low channel correlation & 10.79 & 4.65 \\
Bridge channels & 3.78 & 3.05 \\
All methods & 16.85 & 5.50 \\
Components & & \\
Residual variance & 49.45 & 10.70 \\
Neural components & 44.67 & \\
Biological (nonneural) components & 28.94 & 7.95 \\
\hline
\end{tabular}

and baseline corrected using the -200 to 0 ms prestimulus window. At this step, a final quality check was completed to identify (and remove) channels that had extreme voltage fluctuations $( \pm 50 \mathrm{mV})$. Channels that were flagged during preprocessing were interpolated in order to reconstitute the full montage of 103 channels ( 96 scalp, 7 exogenous) using spherical spline. Similar to previous studies (Hassall, Holland, \& Krigolson, 2013; Kessler et al., 2017), the current study used central midline sites (Cz: electrodes A19 and B19 on our montage) to identify the $\mathrm{P} 3$ activation.

\section{Statistical analyses}

Statistical analyses were performed using STATSLAB, an open-source toolbox that implements robust statistics for analysis of single trial EEG data (Campopiano, van Noordt, \& Segalowitz, 2018). This software uses percentile bootstrap and trimmed means, techniques that are robust to distribution characteristics, such as skew, outliers, uneven tails, and various model assumption violations (Wilcox, 2017).

In STATSLAB, single trial data for channels A19 and B19 were extracted and averaged together. For each subject, the single trial data were resampled, with replacement, to generate a surrogate sampling distribution. The $20 \%$ trimmed mean was taken across trials, at each time point (i.e., removing the most extreme voltages at each time point), to generate a robust bootstrapped ERP. This process was repeated for each condition and the difference taken. Iterating this process of resampling, trimming, and scoring the difference wave was performed 1,000 times to generate a distribution of differences between conditions (see Campopiano, van Noordt, \& 
Segalowitz, 2018 for details). The $95 \%$ confidence interval was obtained to test significant differences between ERP wave forms for each condition. To investigate sensitivity to negative feedback, we ran two 2x2 ANOVAs: (1) worry status (worry vs. low-worry) and grade group (younger vs. older) as the between-subject independent variables, and (2) worry status (worry vs. low-worry) and puberty status (early-pre puberty vs. mid-late puberty) as the between-subject independent variables.

\section{Results}

\section{Descriptive Results}

We used grade (grades 3 to $5=$ children, grades 6 to $8=$ adolescent) and puberty (pre to early puberty = children, mid to late puberty $=$ adolescent) to differentiate between children and adolescents. In order to be consistent with previous research investigating worry and the P3, a median split was used to differentiate between those who had higher versus lower levels of worry (De Pascalis et al. 2004; Bar-ham et al. 2005; Miltner et al., 2005; Reeb-Surtherland et al., 2009). This created four groups based on grade: (1) younger low-worriers (N $=29, M=1.573, S D=0.417)$, (2) younger worriers $(\mathrm{N}=18$, $M=2.954, S D=0.636)$, (3) older low-worriers $(\mathrm{N}=37, M=$ 1.703, $S D=0.483)$, and (4) older worriers $(\mathrm{N}=31, M=3.194$, $S D=0.485$ ); and four groups based on puberty status: (1) preearly puberty low-worriers $(\mathrm{N}=28, M=1.655, S D=0.411)$, (2) pre-early puberty worriers $(\mathrm{N}=12, M=2.958, S D=$ 0.746), (3) mid-late puberty low-worriers $(\mathrm{N}=39, M=$ $1.658, S D=0.498)$, and (4) mid-late puberty worriers $(\mathrm{N}=$ $36, M=3.176, S D=0.461)$.

\section{BART Behavioural Results}

On average, participants received win-feedback on 47.70 trials and loss-feedback on 48.30 trials. There were no group differences in the amount of win-feedback received or in the amount of loss-feedback received, regardless of whether groups were created using grade-level, $\mathrm{F}(3,105)=0.023, p=$ $0.995, \eta_{\mathrm{p}}{ }^{2}=0.001$, or pubertal status, $\mathrm{F}(3,105)=0.152, p=$ $0.928, \eta_{\mathrm{p}}{ }^{2}=0.004$. There also were no differences between the groups on the percent of trials retained after quality control for either wins or losses ( $\mathrm{Ms}=62-66 \%)$, regardless of whether groups were created using grade-level, $\mathrm{F}(3,97)=1.44, p=$ $0.237, \eta p^{2}=0.048$, or pubertal status $\mathrm{F}(3,97)=0.953, p=$ $0.419, \eta \mathrm{p}^{2}=0.033$.

The key variables of interest for the BART behavioural data were: (1) total number of points earned, (2) total number of pumps, (3) reaction time after loss feedback minus reaction time after win feedback (a positive reaction time suggests a longer reaction time to losses compared with wins, whereas a negative reaction time suggests a longer reaction time to wins compared with losses), (4) change in number of pumps (from the previous trial) after a loss, (5) change in number of pumps (from the previous trial) after a win. For each of the outcome variables, two $2 \times 2$ ANOVAs were conducted: (1) with grade (younger vs. older) and worry status (high-worry vs. low-worry) as the independent variables, and (2) with puberty (preearly puberty vs. mid-later puberty) and worry status (highworry vs. low-worry) as the independent variables.

We also assessed whether participants changed the number of pumps they chose based on the feedback from the previous trial. We found that the older age group decreased the number of pumps after receiving win feedback a greater number of times (mean number $=21.266, S D=5.304$ ) compared with the younger age group (mean number $=18.867, S D=5.480$ ), $F(1,105)=4.229, p=0.042, \eta_{\mathrm{p}}{ }^{2}=0.039$. The older age group was more likely to increase their number of pumps following loss feedback $(M=22.688, S D=4.866)$ compared with the younger group $(M=20.222, S D=5.830), F(1,105)=5.451, p$ $=0.021, \eta_{\mathrm{p}}{ }^{2}=0.049$.

The mid-late puberty group increased their number of pumps following loss feedback $(M=22.542, S D=4.930)$ more often than the pre-early puberty group $(M=19.973$, $S D=5.918), F(1,105)=5.451, p=0.021, \eta_{\mathrm{p}}{ }^{2}=0.049$. In addition, we found a significant interaction between pubertal status and worry status on reaction time after loss feedback win feedback, $F(1,105)=5.231, p=0.024, \eta_{\mathrm{p}}{ }^{2}=0.047$. Simple effects analyses revealed that among the mid-later puberty group, there were no differences found between worriers $(M=13.734, S D=202.861)$ and low-worriers $(M=35.400$, $S D=190.806)$; both groups had a longer reaction time to loss feedback than to win feedback, $t(70)=0.467, p=0.642, d=$ 0.110 . Among the early puberty group, there was a significant difference between worriers $(M=92.178, S D=236.983)$ and low-worriers $(M=-281.749, S D=740.428)$ such that the worriers had a longer reaction time after loss feedback (vs. win feedback) than the low-worry group, $t(33.642)=2.311$, $p=0.027, d=0.680$. There were no other significant main effects or interactions for any of the other BART outcome variables.

\section{ERP Results}

We had three main research questions in terms of the ERP data: (1) Do adolescents have a greater neural sensitivity to negative feedback than children (main effect of age group)?; (2) Do worriers have greater neural sensitivity to feedback than low-worriers (main effect of worry)?; (3) Do adolescents worriers and low-worriers show similar sensitivity to negative feedback, and does that differ among children (interaction between worry and age group)? For all three research questions, we conducted analyses first using grade level and then 
again using pubertal status. Results for sensitivity to positive feedback can be found in Supplemental Figure 1.

\section{Analysis Using Puberty Status.}

Do adolescents have greater sensitivity to negative feedback than children? We found a significant main effect of pubertal status, $t(98)=-1.292, p=0.018$, CI $[-0.179$, $-2.473]$. Adolescents (mid-late puberty) had greater sensitivity to negative feedback than children (pre-early puberty status).

Do worriers have greater sensitivity to negative feedback than low-worriers? We found a significant main effect of worry status, $t(98)=-2.989, p<0.001$, CI $[-1.957$, $-4.143]$. Worriers had greater sensitivity to negative feedback than low-worriers.

Do adolescents worriers and low-worriers show similar sensitivity to negative feedback, and does that differ among children (interaction between worry and age group)? We found a significant two-way interaction between worry status (high-worry vs. low-worry) and pubertal status (pre-early vs. mid-late) for negative feedback as indicated by the P3 (see Fig. 1: the nonoverlapping confidence intervals around $300 \mathrm{~ms}$ highlight that the difference between worriers and low-worriers is significantly different among children and adolescents). Specifically, as shown in Fig. 2, worriers had a larger P3 amplitude to negative feedback compared with lowworriers regardless of whether they were children or adolescents. Of note, children and adolescent worriers did not differ on their P3 amplitude to negative feedback (see Fig. 3). The difference between high-worriers and lowworriers, however, was much smaller among adolescents than with children (see interaction Fig. 1).

\section{Analysis Using Grade Level}

Do adolescents have greater sensitivity to negative feedback than children? We found a significant main effect of grade level, $t(98)=-1.639, p<0.001$, CI $[-0.571$, -2.763 ]. Adolescents (older grade) had greater sensitivity to negative feedback than children (younger grade).

Do worriers have greater sensitivity to negative feedback than low-worriers? We found a significant main effect of worry status, $t(98)=-2.890, p<0.001$, CI $[-1.757$, $-3.975]$. Worriers had greater sensitivity to negative feedback than low-worriers.

Do adolescents worriers and low-worriers show similar sensitivity to negative feedback, and does that differ among children (interaction between worry and age group)? We found a significant two-way interaction between worry status (high-worry vs. low-worry) and grade level (younger grade vs. older grade) for negative feedback as indicated by the P3 (see Fig. 1: the nonoverlapping confidence intervals around $300 \mathrm{~ms}$ highlight that the difference between worriers and low-worriers is significantly different among children and adolescents). Specifically, as shown in Fig. 2, worriers had a larger P3 amplitude to negative feedback compared with lowworriers regardless of whether they were children or adolescents. Of note, children and adolescent worriers did not differ on their P3 amplitude to negative feedback (Fig. 3). The difference between high-worriers and lowworriers, however, was much smaller among adolescents than with children (see interaction Fig. 1).

\section{Discussion}

The purpose of the current ERP study was to investigate sensitivity to negative feedback among children and adolescents who are high and low on worry. Current neurodevelopmental models suggest that adolescence is a time of sensitivity to emotionally salient experiences (e.g., sensitivity to negative feedback; Casey, 2015; Somerville et al., 2010; Steinberg, 2008). Our findings provide support for these models by highlighting that adolescents in general had a neural sensitivity to negative feedback. Indeed, even adolescents who were low on worry demonstrated a large P3 response to negative feedback; providing support for adolescents as a sensitivity period for emotionally arousing stimuli (e.g., receiving negative feedback). This finding is corresponds to other research, suggesting that adolescents may be particularly sensitive to "hot" tasks that are emotionally arousing compared with "cold" tasks (Grose-Fifer, Rodrigues, Hoover, \& Zottoli, 2013; Prencipe et al., 2011). Receiving negative feedback appears to be an emotionally salient event. This result also highlights that sensitivity to feedback is not necessarily tied to worry. We also found that both children and adolescents with high levels of worry are sensitive to negative feedback (i.e., have a large P3 amplitude to negative feedback). Of concern, heightened attention towards threatening/negative events has been speculated to play an important role in the development of anxiety (Pérez-Edgar, 2018). Thus, the current study highlights that the P3 may be an important way to identify individuals who have a large physiological reaction to negative feedback. Given that even younger children who were worriers had a large P3 amplitude, the P3 may be a useful tool to identify individuals who have a sensitivity to negative feedback at young ages-perhaps allowing for earlier intervention.

We also were interested in comparing whether our results differed depending on whether grade level or puberty status 


\section{Loss Feedback Interaction Worry - No Worry}

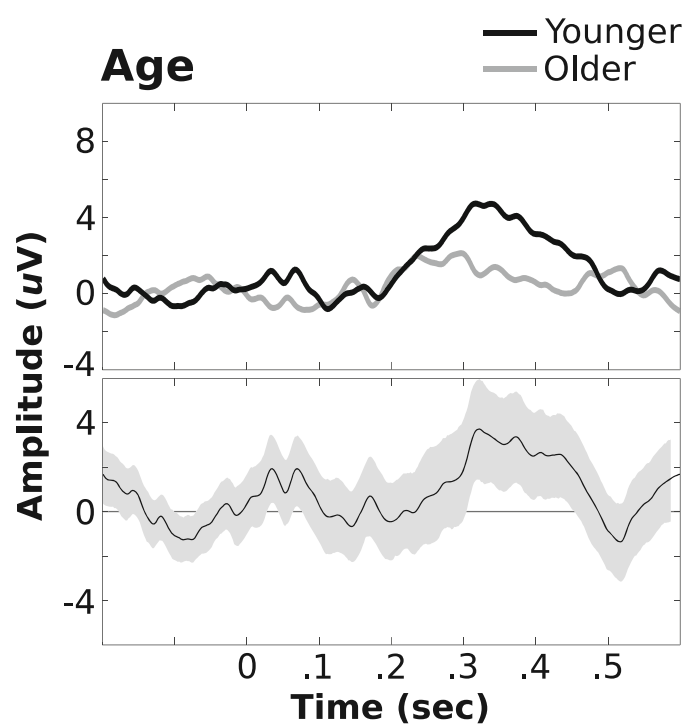

Fig. 1 Loss feedback interaction. Top panels show the difference between worriers and low-worriers for adolescence (grey line) and children (black line). Figures are displayed for both age group (left) puberty group (right). Bottom panels for each figure shows the $95 \%$ bootstrapped
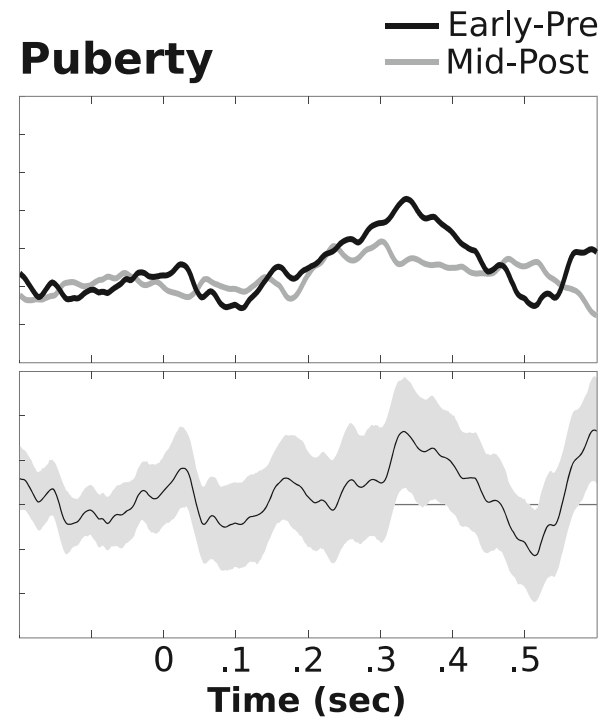

confidence intervals for the difference scores between children and adolescents Confidence intervals not overlapping with the red horizontal line indicate a significant difference at that time point

was used to define adolescence. For the ERP results, our findings remained consistent regardless of the method used to categorize children versus adolescents (Fig. 1). For the behavioural results, there were some consistent findings across methods, but there also were some differences found between using grade level versus puberty status. In terms of the consistent findings, we found that adolescents (either defined by mid-late puberty or older age) were more likely to increase their number of pumps following loss feedback compared with children. This finding might suggest that when adolescents (compared with children) receive losing feedback, they may be more willing to take a risk (e.g., increase their number of pumps), perhaps in an attempt to receive more points to make up for the loss.

In terms of the inconsistent results, we found that adolescents (defined based on older age) were more likely to decrease their number of pumps after receiving win feedback compared with the younger age group. This result was not found when adolescence was defined by pubertal status. It is not entirely clear why the older age group would decrease their number of pumps after a win. It could be that they were trying to protect the points they had just won by using a safer strategy on the following trial.

When adolescence was defined by puberty status, we found a significant interaction between puberty status and worry status on their reaction time after loss feedback-win feedback. Specifically, adolescents and children who were high worriers had a longer reaction time after receiving loss feedback (vs. win feedback) compared with the children nonworriers. In other words, when adolescents and high worriers received negative feedback, they took longer to decide how much to pump the next balloon; thus, they may be taking longer to "recover" from or are more impacted by negative feedback than the children who were low on worry. Of interest, this finding is consistent with the ERP results suggesting that adolescents and high worriers demonstrate a sensitivity to negative feedback. This finding was not significant when adolescence was defined by grade level. Given that the puberty results were more in line with the ERP results, it may suggest that puberty is a better marker of adolescent's attentional bias to negative feedback than age (in line with previous findings; van den Bos, de Rooij, Miers, Bokhorst, \& Westenberg, 2014).

There were no other significant main effects or interactions for any of the other BART outcome variables (e.g., number of pumps). Of note, other ERP studies have failed to find consistent group differences in the BART behavioural outcomes (Kóbor et al., 2015; Takács et al., 2015; Yau et al., 2015). Given that ERP studies often modify the BART task to make it more appropriate to identify ERP components (e.g., include more trials, make stimuli comparable, etc.), these modifications may help to explain why ERP studies are not consistently finding the behavioural results that other non-ERP studies are demonstrating (Lejuez et al., 2007, 2002; White et al., 2008).

In a secondary analysis investigating sensitivity to winfeedback, we found no difference between adolescent worriers and low-worriers. Children with higher levels of worry, however, had a larger neural reaction to positive feedback than children with lower levels of worry. This finding was not 


\section{Loss Feedback}

\section{Children}

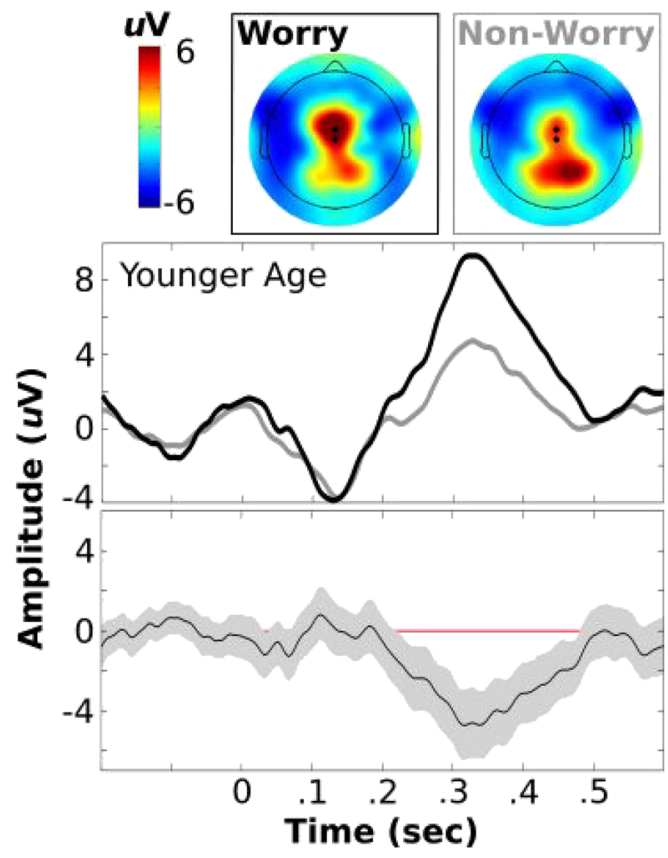

Adolescents
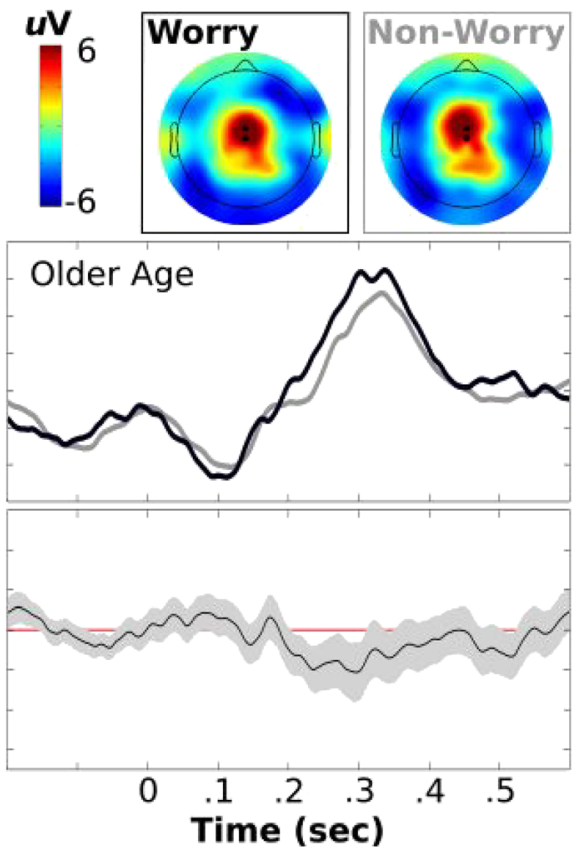

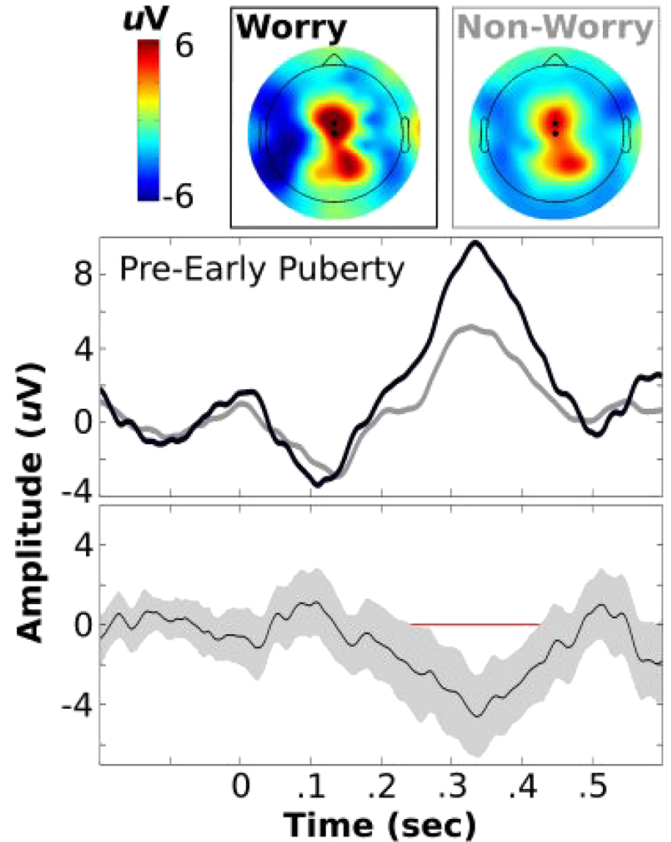

Time (sec)

Fig. 2 Waveforms and topographical maps show the ERPs to loss feedback for worriers and low-worriers separately for both adolescents (right figures) and children (left figures). Figures are displayed for both puberty group (bottom figures) and grade group (top figures). Black dots on topographical maps indicate the channel cluster used for analysis.
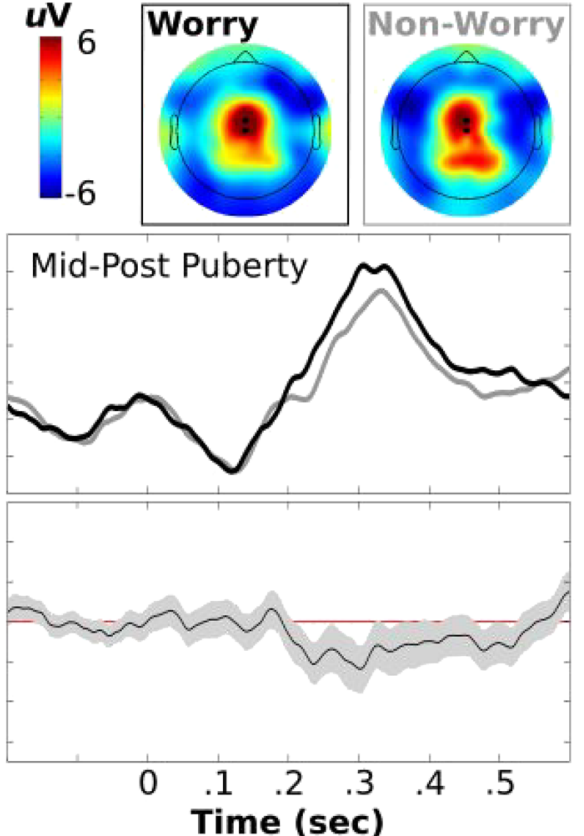

Bottom panels for each figure shows the $95 \%$ bootstrapped confidence intervals for the difference between worriers and low worries [loss for worriers-loss for low worriers]. Confidence intervals that do not overlap with the zero line (red) depict a significant difference at that time point 


\section{Main Effect (Worry)}

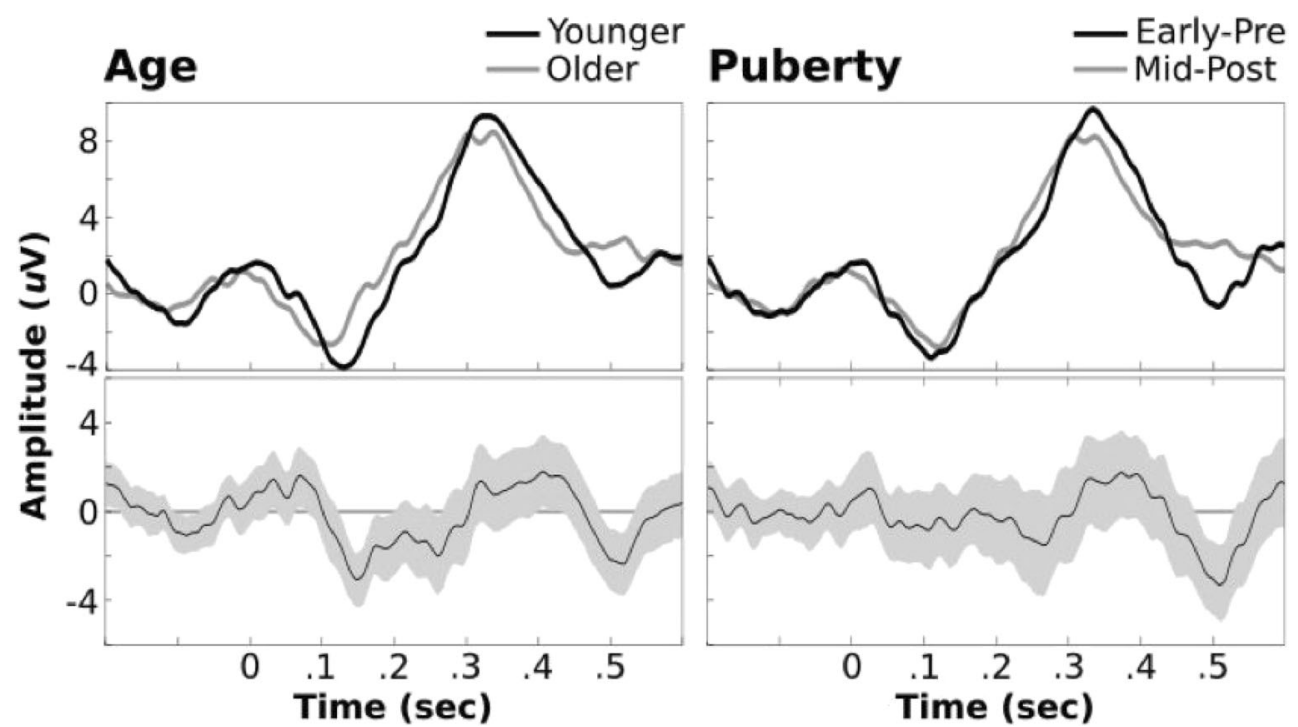

Fig. 3 Results highlighting that children and adolescent worriers were not significantly different—as indicated by the confidence interval overlapping with the zero line at $300 \mathrm{~ms}$

expected and requires further investigation. Of interest, all groups had larger neural sensitivity to negative feedback than to positive feedback - in line with Kahneman and Tversky (1979) who suggested that "losses loom larger than gains."

Despite key strengths of this study, including a large EEG sample and the inclusion of pubertal developmental as indicators of adolescence, the current study is not without limitations. First, we had participants choose the number of pumps they wanted to inflate the balloon at the beginning of the trial. This approach may remove some of the impulsivity involved in pumping up the balloon in real time. Second, our worry measure was a composite of three items as opposed to a complete full-scale worry measure. As the data were part of a larger study assessing a wide range of constructs, it was not feasible to include every item from a worry scale. Of note, however, the alpha for the measure used in this study was 0.838, demonstrating good reliability (Cronbach, 1951; Santos, 1999).

Overall, our findings lend support to theoretical models highlighting that adolescents may be more sensitive to emotionally salient events (e.g., receiving negative feedback) than children. Importantly, we found individual differences in sensitivity to negative feedback; worriers had even greater sensitivity than nonworriers, but this difference was much smaller among adolescents. These findings support current neurodevelopmental models highlighting adolescence as a time of sensitivity to emotionally salient stimuli. Furthermore, our study highlights the importance of investigating individual differences among adolescents and children. Indeed, by separating worriers from nonworriers in both samples, we were able to test whether adolescents in general demonstrate a sensitivity or whether this sensitivity is linked to worry status. Future studies should continue to investigate individual differences among children and adolescents' sensitivity to emotionally salient events as a way of furthering our understanding of adolescent neurodevelopment.

Acknowledgements The second author acknowledges funding for this study received from Canadian Institutes of Health Research.

\section{References}

Balle, M., Tortella-Feliu, M., \& Bornas, X. (2013). Distinguishing youths at risk for anxiety disorders from self-reported BIS sensitivity and its psychophysiological concomitants. International Journal of Psychology https://doi.org/10.1080/00207594.2012.723804

Bar-Haim, Y., Lamy, D., \& Glickman, S. (2005). Attentional bias in anxiety: A behavioral and ERP study. Brain and Cognition, 59(1), 11-22. https://doi.org/10.1016/j.bandc.2005.03.005

Bolling, D. Z., Pitskel, N. B., Deen, B., Crowley, M. J., Mayes, L. C., \& Pelphrey, K. A. (2011). Development of neural systems for processing social exclusion from childhood to adolescence. Developmental Science https://doi.org/10.1111/j.1467-7687.2011.01087.x

Campopiano, A., van Noordt, S. J. R., \& Segalowitz, S. J. (2018). STATSLAB: An open-source EEG toolbox for computing singlesubject effects using robust statistics. Behavioural Brain Research https://doi.org/10.1016/j.bbr.2018.03.025

Carskadon, M. A., \& Acebo, C. (1993). A self-administered rating scale for pubertal development. Journal of Adolescent Health https://doi. org/10.1016/1054-139X(93)90004-9 
Casey, B. (2015). Beyond Simple Models of Self-Control to CircuitBased Accounts of Adolescent Behavior. Ssrn, 295-319. https:// doi.org/10.1146/annurev-psych-010814-015156

Chandrakumar, D., Feuerriegel, D., Bode, S., Grech, M., \& Keage, H. A. D. (2018). Event-related potentials in relation to risk-taking: A systematic review. Frontiers in Behavioral Neuroscience, 12, 111. https://doi.org/10.3389/fnbeh.2018.00111

Cronbach, L. J. (1951). Coefficient alpha and the internal structure of tests. Psychometrika 16, 297-334. https://doi.org/10.1007/ BF02310555

Delorme, A., \& Makeig, S. (2004). EEGLAB: an open source toolbox for analysis of single-trial EEG dynamics including independent component analysis. Journal of Neuroscience Methods. https://doi.org/ 10.1016/j.jneumeth.2003.10.009

Desjardins, J. A., \& Segalowitz, S. J. (2013). Deconstructing the early visual electrocortical responses to face and house stimuli. Journal of Vision, 13(5), 1-18. https://doi.org/10.1167/13.5.22.doi

De Pascalis, V., Strippoli, E., Riccardi, P., \& Vergari, F. (2004). Personality, event-related potential (ERP) and heart rate (HR) in emotional word processing. Personality and Individual Differences, 36(4), 873-891. https://doi.org/10.1016/S01918869(03)00159-4

Euser, A. S., Evans, B. E., Greaves-Lord, K., Huizink, A. C., \& Franken, I. H. A. (2013). Parental rearing behavior prospectively predicts adolescents' risky decision-making and feedback-related electrical brain activity. Developmental Science, 16(3), 409-427. https://doi. org/10.1111/desc. 12026

Fein, G., \& Chang, M. (2008). Smaller feedback ERN amplitudes during the BART are associated with a greater family history density of alcohol problems in treatment-naïve alcoholics. Drug and Alcohol Dependence, 92(1-3), 141-148. https://doi.org/10.1016/j. drugalcdep.2007.07.017

Galvan, A., Hare, T. A., Parra, C. E., Penn, J., Voss, H., Glover, G., \& Casey, B. J. (2006). Earlier Development of the Accumbens Relative to Orbitofrontal Cortex Might Underlie Risk-Taking Behavior in Adolescents. Journal of Neuroscience, 26(25), 68856892. https://doi.org/10.1523/JNEUROSCI.1062-06.2006

Goddings, A. L., Mills, K. L., Clasen, L. S., Giedd, J. N., Viner, R. M., \& Blakemore, S. J. (2014). The influence of puberty on subcortical brain development. NeuroImage. https://doi.org/10.1016/j. neuroimage.2013.09.073

Gogtay, N., Giedd, J. N., Lusk, L., Hayashi, K. M., Greenstein, D., Vaituzis, A. C., ... Thompson, P. M. (2004). Dynamic mapping of human cortical development during childhood through early adulthood. Proceedings of the National Academy of Sciences of the United States of America, 101(21), 8174-8179. https://doi.org/10. 1073/pnas.0402680101

Grose-Fifer, J., Rodrigues, A., Hoover, S., \& Zottoli, T. (2013). Attentional capture by emotional faces in adolescence. Advances in Cognitive Psychology. https://doi.org/10.2478/v10053-0080134-9

Gu, R., Zhang, D., Luo, Y., Wang, H., \& Broster, L. S. (2018). Predicting risk decisions in a modified Balloon Analogue Risk Task: Conventional and single-trial ERP analyses. Cognitive, Affective and Behavioral Neuroscience, 18(1), 99-116. https://doi.org/10. 3758/s13415-017-0555-3

Hare, T. A., Tottenham, N., Galvan, A., Voss, H. U., Glover, G. H., \& Casey, B. J. (2008). Biological substrates of emotional reactivity and regulation in adolescence during an emotional go-nogo task. Biological Psychiatry, 63(10), 927-934. https://doi.org/10.1016/j. biopsych.2008.03.015

Hassall, C. D., Holland, K., \& Krigolson, O. E. (2013). What do I do now? An electroencephalographic investigation of the explore/ exploit dilemma. Neuroscience, 228, 361-370. https://doi.org/10. 1016/j.neuroscience.2012.10.040
Huang, W.-J., Chen, W.-W., \& Zhang, X. (2015). The neurophysiology of P 300-an integrated review. European Review for Medical and Pharmacological Sciences, 19(8), 1480-1488.

Kessler, L., Hewig, J., Weichold, K., Silbereisen, R. K., \& Miltner, W. H. R. (2017). Feedback negativity and decision-making behavior in the Balloon Analogue Risk Task (BART) in adolescents is modulated by peer presence. Psychophysiology, 54(2), 260-269. https://doi. org/10.1111/psyp. 12783

Kiat, J., Straley, E., \& Cheadle, J. E. (2016). Escalating risk and the moderating effect of resistance to peer influence on the P200 and feedback-related negativity. Social Cognitive and Affective Neuroscience, 11(3), 377-386. https://doi.org/10.1093/scan/nsv121

Kóbor, A., Takács, Á., Janacsek, K., Németh, D., Honbolygó, F., \& Csépe, V. (2015). Different strategies underlying uncertain decision making: Higher executive performance is associated with enhanced feedback-related negativity. Psychophysiology, 52(3), 367-377. https://doi.org/10.1111/psyp.12331

Kahneman, D., \& Tversky, A. (1979). Prospect Theory: An analysis of decision under risk. Econometrica: Journal of the Econometric Society, 47(2), 263-291. https://doi.org/10.2307/1914185

Lejuez, C. W., Aklin, W., Daughters, S., Zvolensky, M., Kahler, C., \& Gwadz, M. (2007). Reliability and validity of the youth version of the Balloon Analogue Risk Task (BART-Y) in the assessment of risk-taking behavior among inner-city adolescents. Journal of Clinical Child and Adolescent Psychology, 36(1), 106-111. https:// doi.org/10.1080/15374410709336573

Lejuez, C. W., Richards, J. B., Read, J. P., Kahler, C. W., Ramsey, S. E., Stuart, G. L., ... Brown, R. A. (2002). Evaluation of a behavioral measure of risk taking: The balloon analogue risk task (BART). Journal of Experimental Psychology: Applied, 8(2), 75-84. https:// doi.org/10.1037/1076-898X.8.2.75

Luck, SJ. (2005). An introduction to event related potentials and their neural origins. An Introduction to the Event Related Potential Technique, 11. https://ci.nii.ac.jp/naid/10030430963/

Mills, K. L., Goddings, A. L., Clasen, L. S., Giedd, J. N., \& Blakemore, S. J. (2014). The developmental mismatch in structural brain maturation during adolescence. Developmental Neuroscience, 36(3-4), 147-160. https://doi.org/10.1159/000362328

Miltner, W. H. R., Trippe, R. H., Krieschel, S., Gutberlet, I., Hecht, H., \& Weiss, T. (2005). Event-related brain potentials and affective responses to threat in spider/snake-phobic and non-phobic subjects. International Journal of Psychophysiology, 57(1), 43-52. https:// doi.org/10.1016/j.jpsycho.2005.01.012

Moor, B. G., van Leijenhorst, L., Rombouts, S. A. R. B., Crone, E. A., \& van der Molen, M. W. (2010). Do you like me? Neural correlates of social evaluation and developmental trajectories. Social Neuroscience, 5(5), 461-482. https://doi.org/10.1080/ 17470910903526155

O’Brien, S. F., \& Bierman, K. L. (1988). Conceptions and perceived influence of peer groups: interviews with preadolescents and adolescents. Child Development. https://doi.org/10.1111/j.1467-8624. 1988.tb01504.x

Oostenveld, R., Fries, P., Maris, E., \& Schoffelen, J.-M. (2011). FieldTrip: Open source software for advanced analysis of MEG, EEG, and invasive electrophysiological data. Computational Intelligence and Neuroscience. https://doi.org/10.1155/2011/ 156869

Pérez-Edgar, K. (2018). Attention Mechanisms in Behavioral Inhibition: Exploring and Exploiting the Environment. In K. Pérez-Edgar \& N. A. Fox (Eds.), Behavioral Inhibition: Integrating Theory, Research, and Clinical Perspectives (pp. 237-261). Cham: Springer International Publishing. https://doi.org/10.1007/978-3-319-980775_11

Petersen, A. C., Crockett, L., Richards, M., \& Boxer, A. (1988). A selfreport measure of pubertal status: Reliability, validity, and initial 
norms. Journal of Youth and Adolescence https://doi.org/10.1007/ BF01537962

Pleskac, T. J., Wallsten, T. S., Wang, P., \& Lejuez, C. W. (2008). Development of an Automatic Response Mode to Improve the Clinical Utility of Sequential Risk-Taking Tasks. Experimental and Clinical Psychopharmacology, 16(6), 555-564. https://doi. org/10.1037/a0014245

Prencipe, A., Kesek, A., Cohen, J., Lamm, C., Lewis, M. D., \& Zelazo, P. D. (2011). Development of hot and cool executive function during the transition to adolescence. Journal of Experimental Child Psychology, 108(3), 621-637. https://doi.org/10.1016/j.jecp.2010. 09.008

Reeb-Sutherland, B. C., Vanderwert, R. E., Degnan, K. A., Marshall, P. J., Pérez-Edgar, K., Chronis-Tuscano, A., ... Fox, N. A. (2009). Attention to novelty in behaviorally inhibited adolescents moderates risk for anxiety. Journal of Child Psychology and Psychiatry and Allied Disciplines, 50(11), 1365-1372. https://doi.org/10.1111/j. 1469-7610.2009.02170.x

Sewell, C., Palermo, R., Atkinson, C., \& McArthur, G. (2008). Anxiety and the neural processing of threat in faces. NeuroReport. https:// doi.org/10.1097/WNR.0b013e32830baadf

Somerville, L. H., Jones, R. M., \& Casey, B. J. (2010). A time of change: Behavioral and neural correlates of adolescent sensitivity to appetitive and aversive environmental cues. Brain and Cognition, 72(1), 124-133. https://doi.org/10.1016/j.bandc.2009.07.003

Spear, L. P. (2000). The adolescent brain and age-related behavioral manifestations. Neuroscience and Biobehavioral Reviews.

Steinberg, L. (2008). A social neuroscience perspective on adolescent risk-taking. Developmental Review, 28(1), 78-106. https://doi.org/ 10.1016/j.dr.2007.08.002

Santos, J. R. A. (1999). Cronbach's alpha: A tool for assessing the reliability of scales. Journal of extension, 37(2), 1-5.

Takács, Á., Kóbor, A., Janacsek, K., Honbolygó, F., Csépe, V., \& Németh, D. (2015). High trait anxiety is associated with attenuated feedback-related negativity in risky decision making. Neuroscience Letters, 600, 188-192. https://doi.org/10.1016/j.neulet.2015.06.022

van den Bos, E., de Rooij, M., Miers, A. C., Bokhorst, C. L., \& Westenberg, P. M. (2014). Adolescents' increasing stress response to social evaluation: pubertal effects on cortisol and alpha-amylase during public speaking. Child Development, 85(1), 220-236. https:// doi.org/10.1111/cdev.12118

van Noordt, S. J. R., Desjardins, J. A., Gogo, C. E. T., Tekok-Kilic, A., \& Segalowitz, S. J. (2017). Cognitive control in the eye of the beholder: Electrocortical theta and alpha modulation during response preparation in a cued saccade task. NeuroImage. https://doi.org/10. 1016/j.neuroimage.2016.09.054

van Noordt, S. J. R., Desjardins, J. A., \& Segalowitz, S. J. (2015). Watch out! Medial frontal cortex is activated by cues signaling potential changes in response demands. NeuroImage, 114, 356-370. https:// doi.org/10.1016/j.neuroimage.2015.04.021

Vervoort, L., Wolters, L. H., Hogendoorn, S. M., de Haan, E., Boer, F., \& Prins, P. J. M. (2010). Sensitivity of Gray's Behavioral Inhibition System in clinically anxious and non-anxious children and adolescents. Personality and Individual Differences. https://doi.org/10. 1016/j.paid.2009.12.021

Webber, T. A., Soder, H. E., Potts, G. F., Park, J. Y., \& Bornovalova, M. A. (2017). Neural outcome processing of peer-influenced risk-taking behavior in late adolescence: Preliminary evidence for gene $x$ environment interactions. Experimental and Clinical Psychopharmacology, 25(1), 31-40. https://doi.org/10.1037/ pha0000105

Westenberg, P. M., Drewes, M. J., Goedhart, A. W., Siebelink, B. M., \& Treffers, P. D. A. (2004). A developmental analysis of self-reported fears in late childhood through mid-adolescence: Social-evaluative fears on the rise? Journal of Child Psychology and Psychiatry and Allied Disciplines https://doi.org/10.1111/j.1469-7610.2004.00239. $\mathrm{x}$

White, T. L., Lejuez, C. W., \& de Wit, H. (2008). Test-retest characteristics of the Balloon Analogue Risk Task (BART). Experimental and Clinical Psychopharmacology, 16(6), 565-570. https://doi.org/10. 1037/a0014083

Wilcox, R. R. (2017). Introduction to Robust Estimation and Hypothesis Testing. Introduction to Robust Estimation and Hypothesis Testing (4th). San Diego, CA: Academic Press. https://doi.org/10.1016/ C2010-0-67044-1

Xu, S., Pan, Y., Wang, Y., Spaeth, A. M., Qu, Z., \& Rao, H. (2016). Real and hypothetical monetary rewards modulate risk taking in the brain. Scientific Reports, 6(June), 1-7. https://doi.org/10.1038/ srep29520

Yau, Y. H. C., Potenza, M. N., Mayes, L. C., \& Crowley, M. J. (2015). Blunted feedback processing during risk-taking in adolescents with features of problematic Internet use. Addictive Behaviors, 45, 156163. https://doi.org/10.1016/j.addbeh.2015.01.008

Publisher's note Springer Nature remains neutral with regard to jurisdictional claims in published maps and institutional affiliations. 